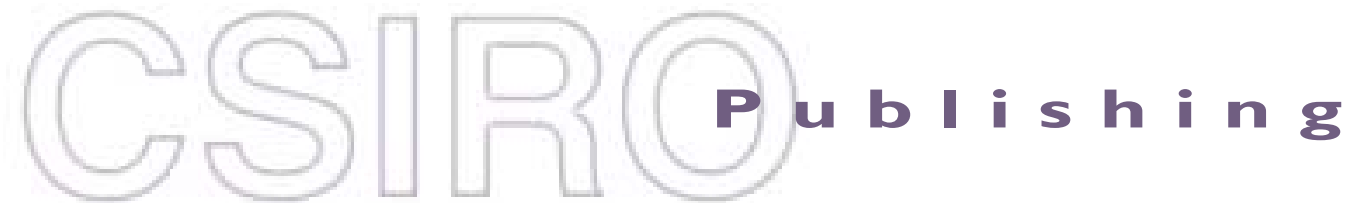

\section{Publications of the Astronomical Society of Australia}

Volume 19, 2002

(C) Astronomical Society of Australia 2002

An international journal of astronomy and astrophysics

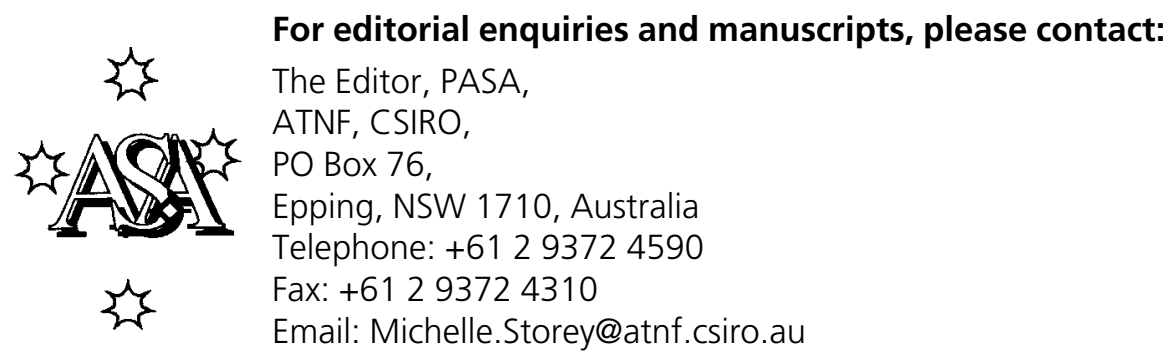

For general enquiries and subscriptions, please contact: CSIRO Publishing PO Box 1139 (150 Oxford St)

Collingwood, Vic. 3066, Australia

Telephone: +6139662 7666

Fax: +61 396627555

Email: publishing.pasa@csiro.au

C S I RO

PUBLISHING Published by CSIRO Publishing

for the Astronomical Society of Australia

www.publish.csiro.au/journals/pasa 


\title{
AGN Variability from the USNO Radio Reference Frame Image Database
}

\author{
Alan L. Fey \\ US Naval Observatory, 3450 Massachusetts Ave NW, Washington, DC 20392, USA \\ afey@usno.navy.mil
}

Received 2001 July 26, accepted 2001 November 5

\begin{abstract}
We present results from preliminary analysis of radio interferometric data taken from the US Naval Observatory Radio Reference Frame Image Database. The results presented here reveal valuable insight into the structural variability of the two extragalactic radio sources $0552+398$ and $1308+326$. We are actively pursuing analysis of additional objects.
\end{abstract}

Keywords: quasars: general — quasars: individual $(0552+398,1308+326)$

\section{Introduction}

The US Naval Observatory (USNO) Radio Reference Frame Image Database (RRFID) is a collection of more than 2700 VLBI 'snapshot' images of over 445 compact extragalactic radio sources (quasars, BL Lac objects, etc.). Images are obtained from Very Long Baseline Array $(\text { VLBA })^{1}$ observations and/or from geodetic/astrometric observations primarily at frequencies of $2 \mathrm{GHz}$ and $8 \mathrm{GHz}$. These data are the result of an ongoing program to image International Celestial Reference Frame (ICRF) (Ma et al. 1998) sources on a regular basis. The ICRF is the IAU sanctioned fundamental celestial position reference system and is currently defined by the radio positions of 212 extragalactic objects. Approximately 50-70 ICRF sources are observed about every two months. Although not all data have been calibrated and reduced, there are sufficient data available for about 70 sources to do structural variability analysis over a two year period with approximately 2 month time sampling. We present preliminary results from variability studies we have undertaken using RRFID data on the sources $0552+398$ and $1308+326$. Because we believe that the RRFID data are a valuable resource, we make these data available for scientific use by anyone. Contour plots of images and plots of visibility data are available directly from our World Wide Web site at http: //www. usno.navy.mil/RRFID/. Images and/or visibility data in FITS format can also be obtained upon request.

\section{Variability of AGN - A Work in Progress}

We first present a 'quick and dirty' method for evaluating the temporal variability of radio frequency intrinsic structure of well observed extragalactic sources in the RRFID. A total of 72 ICRF sources are observed often enough (roughly once every two months over a two

\footnotetext{
${ }^{1}$ The VLBA is a facility of the National Radio Astronomy Observatory (NRAO) which is operated by Associated Universities, Inc., under cooperative agreement with the National Science Foundation.
}

year time period) to warrant a detailed structure analysis. In Section 3 below, we present preliminary results using model fitting for two sources, $0552+398$ and $1308+326$. Because model fitting is an extremely time consuming endeavour, we would like to find a method for quickly quantifying the variability of these sources. We define

$$
I=\int_{0}^{\infty}[1-H(\theta)] d \theta
$$

where $H(\theta)$ is the fraction of the total source flux density contained in a radius $\theta$, which is an angular dimension normalised by the synthesised beam at a particular epoch, centred on the peak of emission. This quantity is easily calculated using the CLEAN components from the final hybrid images. For multiple epoch images, the mean and standard deviation of the mean of quantity $I$ can be calculated. However, because the quantity $I$ appears to be somewhat dependent on parameters of the imaging and heavily dependent on image quality, we are actively searching for other methods to calculate the quantity $I$, such as by using the visibility data rather than the CLEAN components models. As such, the quantity $I$ cannot currently be used for comparison with other astrophysical quantities of interest (such as flux density variability). This is a work in progress.

\section{Detailed Variability Analysis of Two AGN}

\section{$3.10552+398$}

The quasar $0552+398$ is at a redshift of 2.365 ( 1 mas $\approx$ $\left.5.239 \mathrm{pc} ; H_{0}=75 \mathrm{~km} \mathrm{~s}^{-1} \mathrm{Mpc}^{-1}, q_{0}=0.5\right)$. We have analysed RRFID data from 17 epochs by fitting circular Gaussian model components to the self-calibrated visibility data (see Fey \& Charlot 2000 and references therein for an explanation of the procedures used to calibrate and image the RRFID data). At an observing frequency of $2.3 \mathrm{GHz}$, the source can be represented at all observed epochs by a circular Gaussian of width $\approx 1$ mas. At $8.6 \mathrm{GHz}$, the source consists of two closely spaced components with an approximately constant separation 


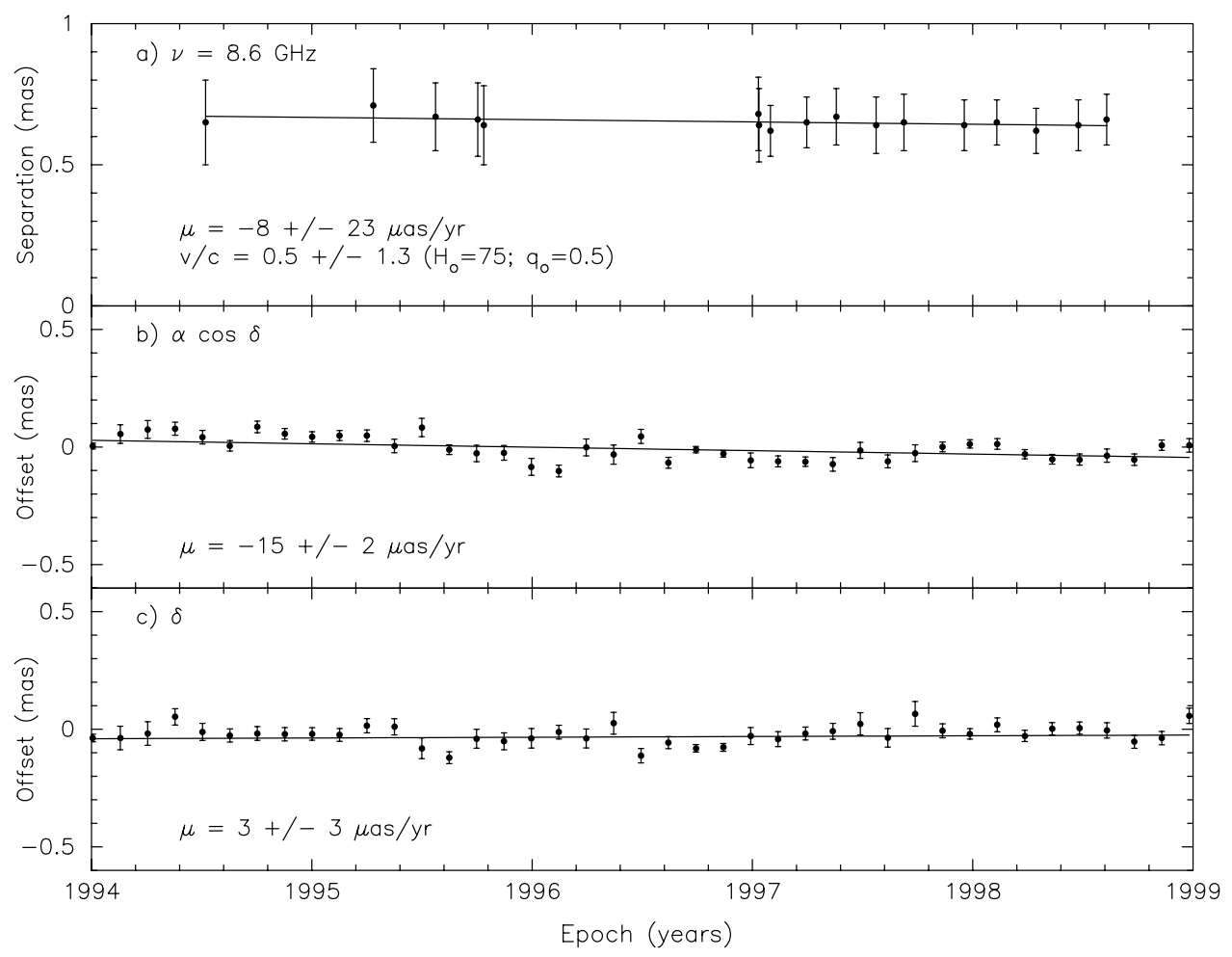

Figure 1 (a) Core-jet component separation for $0552+398$ at $8.6 \mathrm{GHz}$; (b) position time series in right ascension; (c) position time series in declination. Position time series are shown as an offset from a mean position. Solid lines represent least squares fits to a straight line.

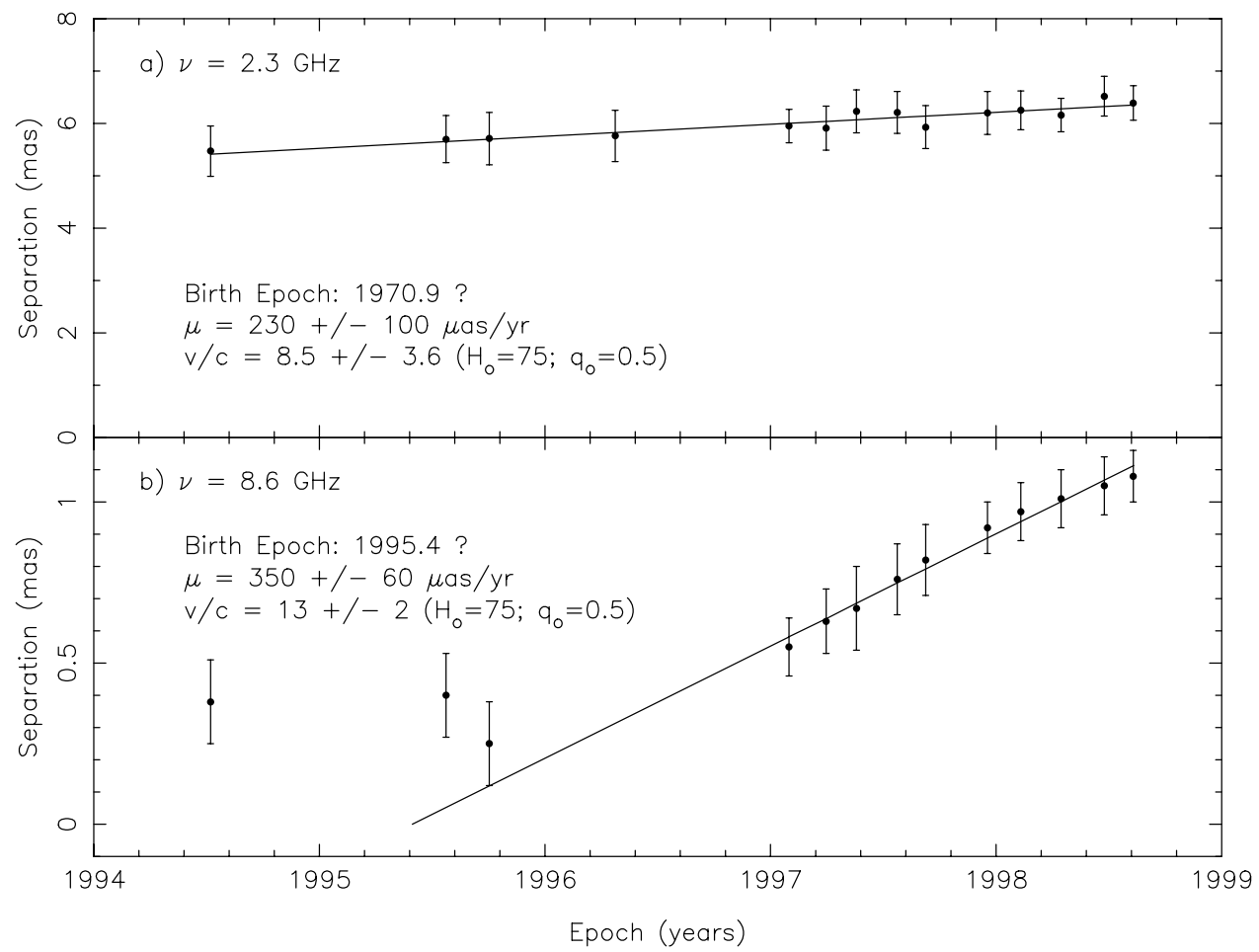

Figure 2 Core-jet component separation for $1308+326$ at (a) $2.3 \mathrm{GHz}$, and (b) $8.6 \mathrm{GHz}$. Solid lines represent least squares fits to a straight line.

of $\approx 0.65$ mas in the east-west direction. The component separation at $8.6 \mathrm{GHz}$ is shown in Figure 1a for 17 epochs spanning approximately five years. Shown in Figure $1 \mathrm{~b}$ and $1 \mathrm{c}$ are the position time series in right ascension and declination taken from an astrometric VLBI solution using the USNO geodetic database (see Fey, Eubanks, \& Kingham 1997 and references therein for an explanation of the analysis used to estimate source 


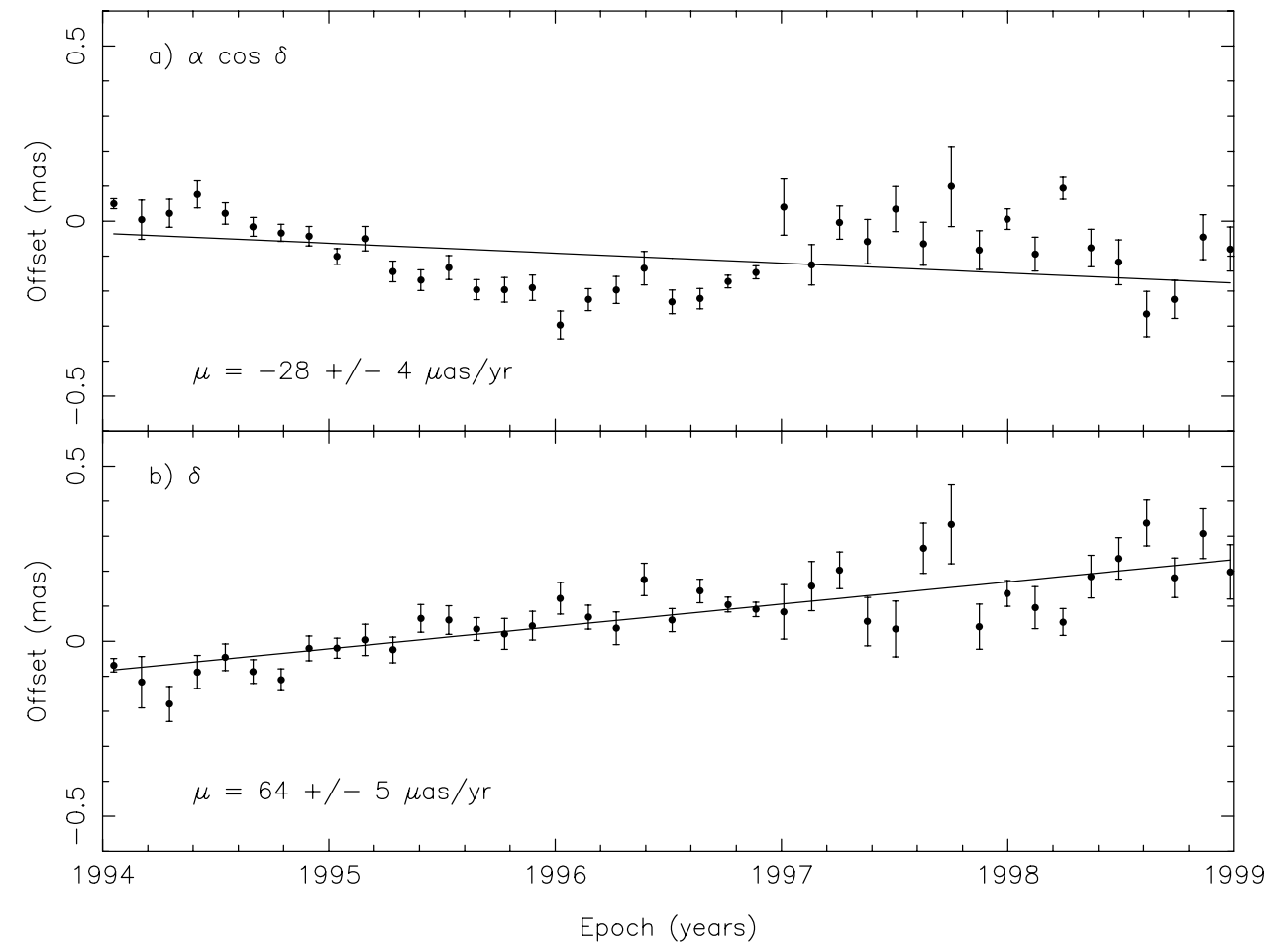

Figure 3 (a) Position time series in right ascension for 1308+326; (b) position time series in declination. Position time series are shown as an offset from a mean position. Solid lines represent least squares fits to a straight line.

proper motions). As can be seen, the source $0552+398$ is a compact and remarkably stable source with only small structure variations $(\sim 20 \mu$ as rms variations about an approximately constant separation) and only a small proper motion in right ascension $\left(\sim 15 \mu \mathrm{as} \mathrm{yr}^{-1}\right)$ as seen in the position time series. The apparent proper motion seen in the position time series is presumably due to intrinsic structure variations but could possibly be due to a residual frame rotation in the astrometric solution. The stability of the source is supported by the lack of significant flux density variability over the concurrent five year period.

\section{$3.21308+326$}

The quasar $1308+326$ is at a redshift of $0.997(1$ mas $\approx$ $5.674 \mathrm{pc}$ ). We have analysed RRFID data from 14 epochs by fitting circular Gaussian model components to the selfcalibrated visibility data. At both observing frequencies of 2.3 and $8.6 \mathrm{GHz}$, the source can be well represented by two compact components which are separating superluminally at apparent velocities of $8.5 c$ and $13 c$, respectively, in roughly the east-west direction. The component separations at 2.3 and $8.6 \mathrm{GHz}$ are shown in Figure 2 for 14 epochs spanning approximately five years. The birth of the $8.6 \mathrm{GHz}$ superluminal component at epoch $\sim 1995.4$ appears to have been associated with a flare in the source flux density which peaked during the time period between 1995 and 1996. There is some evidence from the images to suggest that the $8.6 \mathrm{GHz}$ component was ejected earlier than 1995.4 with a smaller superluminal velocity and was then accelerated between 1996 and 1997. This is supported by the fact that the astrometric position time series (Figure 3) shows motion of the source well before the extrapolated birth epoch of the $8.6 \mathrm{GHz}$ component. The most recent imaging data suggests that the $8.6 \mathrm{GHz}$ component is currently decelerating. This is supported by the fact that the $2.3 \mathrm{GHz}$ component, which has a greater projected separation from the core than the $8.6 \mathrm{GHz}$ component, has a smaller superluminal velocity suggesting that it also has decelerated (assuming it also was initially ejected at a higher velocity). As can be seen, the source $1308+326$ is a compact but remarkably complex source with significant structure variation, significant flux density variation, and high apparent proper motion in both right ascension and declination. This source was originally classified as an ICRF defining source but has recently been downgraded in the most recent update of the ICRF (IERS 1999).

\section{References}

Fey, A. L., \& Charlot, P. 2000, ApJS, 128, 17

Fey, A. L., Eubanks, T. M., \& Kingham, K. A. 1997, AJ, 114, 2284

IERS 1999: 1998 International Earth Rotation Service Annual Report, ed. D. Gambis, Observatoire de Paris, pp. 87-114

Ma, C., et al. 1998, AJ, 116, 516 